\title{
Characterization of the Brassica campestris mitochondrial gene for subunit six of NADH dehydrogenase: nad6 is present in the mitochondrion of a wide range of flowering plants
}

\author{
Jacqueline M. Nugent * and Jeffrey D. Palmer \\ Department of Biology, Indiana University, Bloomington, IN 47405, USA \\ Received May 8, 1992/Accepted June 25, 1992
}

\begin{abstract}
We have isolated the Brassica campestris mitochondrial gene nad6, coding for subunit six of NADH dehydrogenase. The deduced amino-acid sequence of this gene shows considerable similarity to mitochondrially encoded NAD6 proteins of other organisms as well as to NAD6 proteins coded for by plant chloroplast DNAs. The B. campestris nad6 gene appears to lack introns and produces an abundant transcript which is comparable in size to a previously described, unidentified transcript (\#18) mapped to the $B$. campestris mitochondrial genome. An alignment of NAD6 proteins (deduced from DNA sequences) suggests that $B$. campestris nad6 transcripts are edited. Southern-blot hybridization indicates that nad6 is present in the mitochondrial genome of all of a wide range of flowering plant species examined.
\end{abstract}

Key words: $n a d 6$ - Flowering plants - Mitochondrial gene

\section{Introduction}

NADH: ubiquinone reductase (NADH dehydrogenase, complex I) is a multimeric inner mitochondrial membrane enzyme that forms the link between the Krebs cycle and the electron transport chain (Moller 1986). This enzyme is very complex and contains about 30 different protein subunits. The genes coding for these subunits are partitioned between the nuclear and mitochondrial genomes of most organisms. Surprisingly, genes coding for 11 subunits of NADH dehydrogenase have also been found in land plant chloroplast genomes (Berger et al. 1991; Dupuis et al. 1991), suggesting that chloroplasts contain an NADH dehydrogenase that may be involved in a chloro-respiratory pathway.

The completely sequenced mitochondrial genomes of the land plant Marchantia polymorpha (Oda et al. 1992),

* Present address: John Innes Institute, Colney Lane, Norwich NR4 7UH, UK

Correspondence to: J. D. Palmer the filamentous ascomycete Podospora anserina (Cummings et al. 1990) and a variety of animals (reviewed by Attardi and Schatz 1988) contain genes that specify the same seven subunits of NADH dehydrogenase (subunits 1, 2, 3, 4, 4L, 5 and 6). Mitochondrial DNAs (mtDNAs) of other organisms contain a subset of these genes or additional genes for other subunits of complex I. The Chlamydomonas reinhardtii mitochondrial genome contains genes for subunits 1, 2, 4, 5, and 6 (Michaelis et al. 1990); Paramecium mtDNA codes for subunits 1, 2, 3, 4, 5, 7, and 9 (Pritchard et al. 1990); and Trypanosoma brucii mtDNA encodes, at a minimum, subunits $1,4,5,7$ and 8 (Payne et al. 1985; Jasmer et al. 1985; Feagin and Stuart 1985; Koslowsky et al. 1990; Souza et al. 1992). Notable exceptions are the yeasts Saccharomyces cereviseae and Schizosaccharomyces pombe, whose mitochondrial genomes do not contain any NADH dehydrogenase genes (reviewed by Attardi and Schatz 1988), and whose mitochondria may entirely lack a complex I-like NADH dehydrogenase (deVries and Grivell 1988). So far, seven genes coding for subunits of NADH dehydrogenase have been identified in flowering plant mtDNAs: nad1 (Chapdelaine and Bonen 1991; Wissinger et al. 1991) nad2 (Xue et al. 1990; Binder et al. 1992) nad3 (Gualberto et al. 1988), nad4 (Lamattina and Grienenberger 1991; Gass et al. 1992) nad5 (Knoop et al. 1991), nad7 (L. Bonen personal communication), and $\operatorname{nad} 9$ (J. M. Grienenberger personal communication). However, since flowering plant mtDNAs are by far the largest and most complex mtDNAs known, ranging in size from 208 to $2400 \mathrm{~kb}$ (reviewed by Newton 1988) and being extremely prone to structural rearrangement, determination of the complete gene content and organization of these mtDNAs is still far from complete.

A detailed transcriptional map of the Brassica campestris mitochondrial genome revealed 24 abundant and positionally distinct transcripts longer than 500 nucleotides (Makaroff and Palmer 1987). Fifteen of these abundant transcripts could not be identified as producing any known product (protein or RNA). We have sequenced a region of the $B$. campestris mitochondrial genome that 
produces one of these abundant but heretofore unidentified transcripts (\#18; Makaroff and Palmer 1987). We identify an open reading frame (ORF) which is highly expressed and whose transcript is similar in size to the one mapped by Makaroff and Palmer (1987). This ORF codes for subunit 6 of NADH dehydrogenase by virtue of the similarity of its predicted amino-acid sequence to those of other known nad6 genes. The $B$. campestris nad6 is located just downstream from a virtually intact chloroplast-derived $r b c \mathrm{~L}$ gene.

\section{Materials and methods}

Mitochondrial DNAs were isolated from either green leaves or etiolated seedlings according to the DNase I procedure (Kolodner and Tewari 1972). B. campestris total RNA was prepared from etiolated shoot tissue according to a scaled-up version of the guanidinium isothiocyanate extraction procedure of Hamby et al. (1988). $B$. campestris seed (var. Purple-top white globe) was purchased from W. Atlee Burpee and Co. PA, U.S.A. The Southern-blot hybridization was carried out using standard procedures (Sambrook et al. 1989). The membrane was prehybridized and hybridized at $60^{\circ} \mathrm{C}$ in Blotto solution $(0.5 \%$ nonfat dry milk, $1 \% \mathrm{SDS}, 4 \times \mathrm{SSC}$, preheated to $60^{\circ} \mathrm{C}$ ). After hybridization the blot was washed in $2 \times \mathrm{SSC}, 0.5 \% \mathrm{SDS}$ at $60^{\circ} \mathrm{C}$. RNA was electrophoresed through a $1.2 \%$ agarose gel after denaturation with glyoxal and dimethyl sulfoxide (Sambrook et al. 1989) and transferred to a Zetabind membrane. The filter was prehybridized and hybridized at $60^{\circ} \mathrm{C}$ in $5 \%$ SDS, $10 \%$ dextran sulfate, and $1 \mathrm{M} \mathrm{NaCl}$. Post-hybridization washes were as described above. Hybridization probes were labeled using random hexamer oligonucleotide primers (Sambrook et al. 1989). DNA sequencing was by the dideoxynucleotide chain-termination method (Sanger et al. 1977) using a series of nested deletions created by exonuclease III digestion (Erase-A-Base, Promega). All of the P3.1 clone downstream from the chloroplast-derived $r b c \mathrm{~L}$ gene was sequenced on one strand; the nad6 locus was sequenced completely on both strands. The GenBank/EMBL accession number of this sequence is X68256.

Protein and nucleotide sequences were searched using the GCG sequence-analysis software package, version 7.0, April 1991 (Devereux et al. 1984). Protein sequences were searched through the SwissProt database, release number 17.0 (2/91); nucleotide sequences were searched through the GenBank database, release 67 (3/91). The computer program CLUSTAL was used to align protein sequences.

\section{Results}

\section{Sequence analysis and identification of the nad6 gene}

A transcriptional map of the $218-\mathrm{kb}$ B. campestris mitochondrial genome revealed three transcripts $(2000,1900$ and 800 nucleotides) which hybridize to a cloned $P_{s t} \mathrm{I}$ fragment of $3.1 \mathrm{~kb}$ (P3.1) from this genome (Makaroff and Palmer 1987). The two larger transcripts are products of the chloroplast $r b c \mathrm{~L}$ gene (encoding the large subunit of ribulose biphosphate carboxylase), which hybridize to P3.1 because a virtually intact chloroplastderived $r b c \mathrm{~L}$ gene is contained within this mtDNA fragment (Makaroff and Palmer 1987; Nugent and Palmer 1988, and unpublished). The smallest transcript (\#18 in the scheme of Makaroff and Palmer 1987) was inferred to be a mitochondrial transcript but did not correspond to
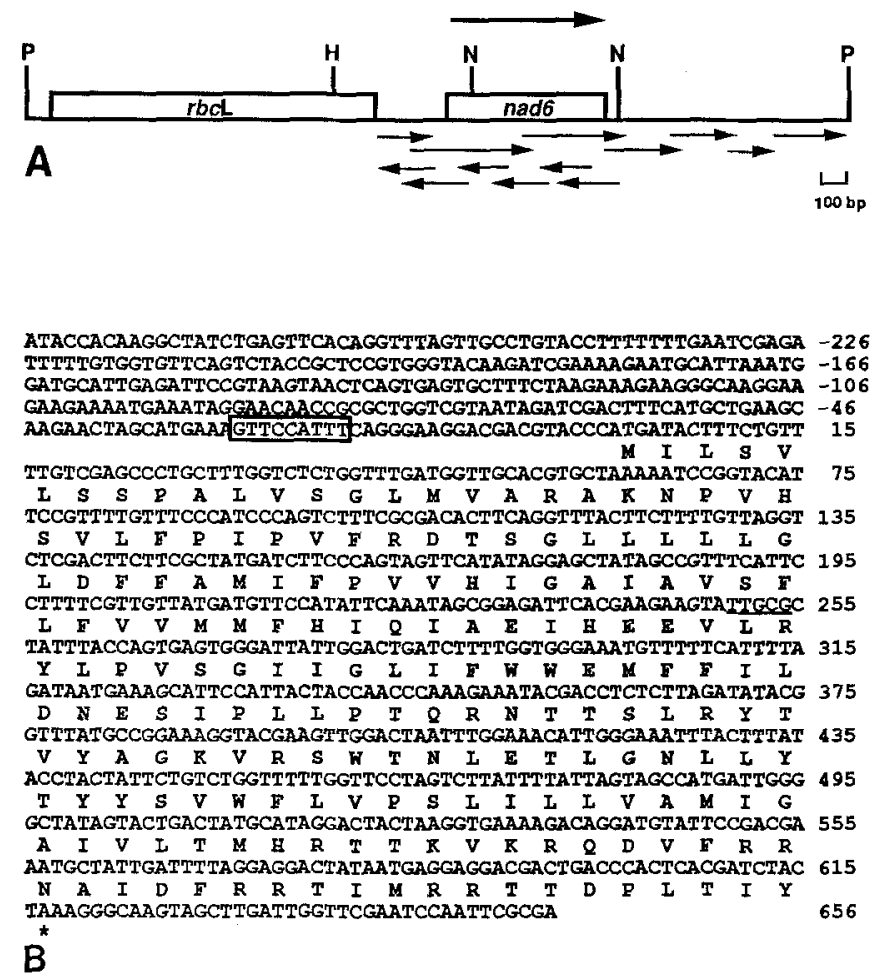

Fig. 1 A, B. Organization (A) and sequence (B) of nad6 in B. campestris mtDNA. A direction of transcription is indicated by the upper arrow: arrows below the drawing indicate the sequencing strategy used in the vicinity of the nad6 gene. Restriction sites mentioned in the text are shown $(P, P s t \mathrm{I} ; H, H i n d I I I ; N, N r u \mathrm{I})$. B the aminoacid translation according to the 'universal' genetic code is shown below the DNA sequence. A putative ribosome-binding site (Fig. 3) upstream of the first in-frame ATG is boxed. Nucleotides $(250-254)$ showing identity to the conserved $5^{\prime}$ splice site of group II A1 introns (Michel et al. 1989) are underlined. The EMBL/GenBank accession number of this sequence is $\mathrm{X} 68256$

any of the known mitochondrial genes in the B. campestris genome (Makaroff and Palmer 1987).

We have sequenced most of the P3.1 clone and present the sequence of a portion of the clone $(941 \mathrm{bp})$ that lies downstream from the chloroplast-derived $r b c L$ gene (Fig. 1). The $r b c \mathrm{~L}$ sequence will be presented in a separate paper on the transfer and evolution of chloroplast $r b c \mathrm{~L}$ genes in plant mitochondrial genomes. The region presented here contains a single ORF of significant size, with the potential to encode a 205 amino-acid polypeptide (Fig. 1B). The best matches between this ORF and sequences in the GenBank nucleotide sequence database and the SwissProt protein sequence database are to mitochondrial and chloroplast nad6 genes and their protein products (Fig. 2). The ends of the nad6 proteins are moderately well conserved in length and sequence, whereas the middle is highly variable in both respects (Fig. 2 and legend to Fig. 2). For example, the B. campestris nad6 is $35 \%$ identical to the $C$. reinhardtii protein over the former's first 78 amino acids and $37 \%$ identical over their last 38 alignable amino acids, whereas the two proteins show no significant similarity over a region corresponding to the central 61 amino acids of the $B$. campestris protein. We conclude that the $B$. campestris ORF is an nad6 gene. 


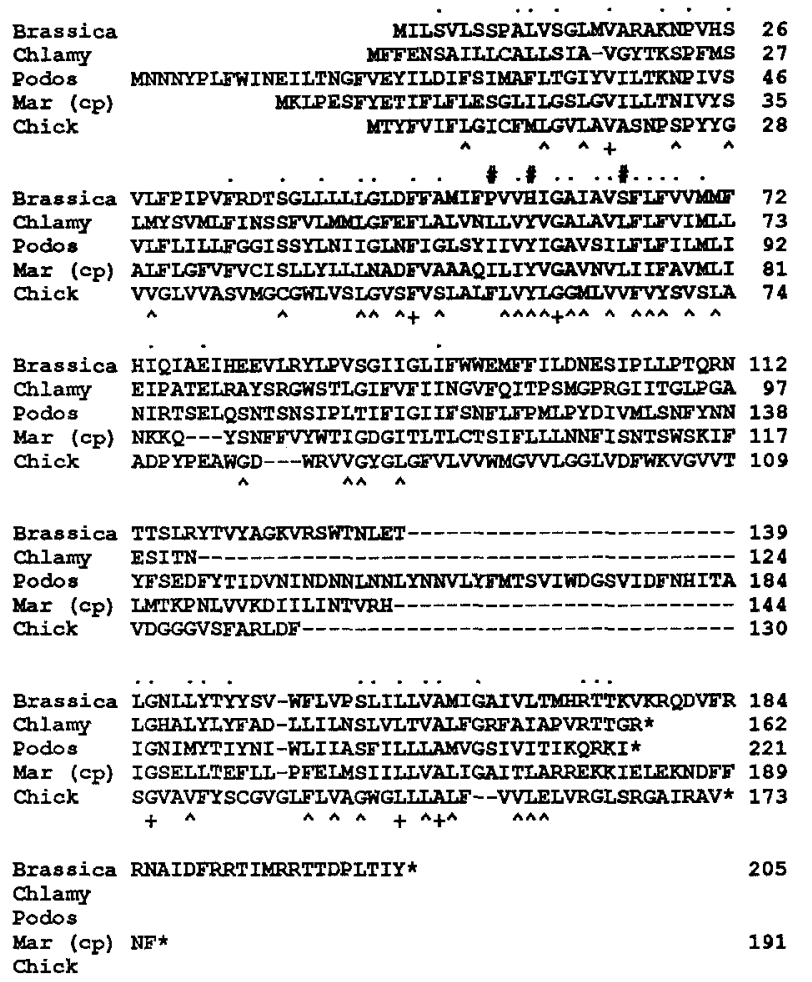

Fig. 2. Alignment of five NAD6 protein sequences. The alignment contains four mitochondrial NAD6 sequences, from $B$. campestris (Brassica; Fig. 1), C. reinhardtii (Chlamy; Boer and Gray 1989), $P$. anserina (Podos; Cummings and Domenico 1988), and chicken (chick; Desjardins and Morais 1990), and the chloroplast NAD6 sequence from $M$. polymorpha [Mar ( $c p$ ); Ohyama et al. 1986]. Three amino-acid positions in the Brassica sequence that may be altered by RNA editing are denoted by '\#'. Gaps are indicated by '-'. Amino acids that are conserved across all five species are indicates by ' + '. Conservative amino-acid changes are indicated by ' $\wedge$ '. Residues that are identical between Brassica and Chlamydomonas are marked by ' $\therefore$. The initial alignment produced by the pairwise, multiple sequence alignment program of CLUSTAL showed a region (corresponding to amino acids 115-184 of Podospora) that was notable for being rich in gaps (three in Brassi$c a$, two different ones in Chlamydomonas, four different ones in Marchantia, and four different ones in chicken) and extremely poorly matched in sequence. In our view, the alignment in this region is completely 'forced', i.e., we cannot discern any evidence of evolutionary homology in it. Accordingly, to reduce the 'gappiness' of the alignment shown, and thereby avoid the implication that the gaps are evolutionarily meaningful, we have deliberately altered the alignment in this region such that all of the amino acids for the four shorter sequences (i.e., all but Podospora) are shown as 'left-justifed', starting at Podospora position 115

This is the first report of a flowering plant mitochondrial gene coding for subunit six of NADH dehydrogenase.

As is the case for several plant mitochondrial genes (MacFarlane et al. 1990), nad6 shows considerable bias towards the use of $\mathrm{A} / \mathrm{T}(62 \%)$ over $\mathrm{G} / \mathrm{C}(38 \%)$ in the third positions of codons. A block of nine nucleotides upstream of the first in-frame AUG of nad6 is identical at seven positions to a region found upstream of the $B$. campestris nad4 gene (Gass et al. 1992) and is complementary at eight of nine positions to the 3 '-terminus of the mitochondrial $18 \mathrm{~s}$ rRNA from Oenothera (Fig. 3).

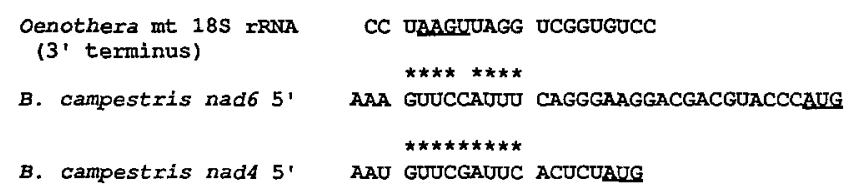

Fig. 3. A putative ribosome binding site is found upstream of the first in-frame AUG (underlined) of nad6. A nononucleotide upstream of the $B$. campestris nad 6 and nad4 genes is complementary to the 3-terminus of Oenothera $18 \mathrm{~s}$ mitochondrial rRNA (allowing for G-U pairing). Nucleotides upstream of nad6 and nad4 that are complementary to the $3^{\prime}$-terminus of $18 s$ rRNA are indicated by stars. An $18 s$ rRNA-sequence (5'-UGAA) complementary to putative 'Shine-Dalgarno' type sequences (Dawson et al. 1984) of other plant mitochondrial mRNAs is underlined. The $18 \mathrm{~s}$ rRNA sequence is from Brennicke et al. (1985); the nad4 sequence is from Gass et al. (1992)

Therefore, we propose that this region may serve as a ribosome binding site. The $B$. campestris sequence compares well with other putative plant mitochondrial ribosome binding sites (Dawson et al. 1984), both in sequence and in position (20 nucleotides) from the presumed start site of translation. However, it should be stressed that no experimental studies have yet been carried out to define functional ribosome binding sites in plant mitochondrial mRNAs and that some plant mitochondrial genes lack any homolog of the proposed ribosome binding site (Lonsdale 1989).

\section{Transcription of the nad6 gene}

A 564-bp NruI fragment, which contains most of the $B$. campestris nad6 locus (Fig. 1A), was used to probe a Northern blot of B. campestris total RNA. A single abundant transcript of approximately $750 \mathrm{nt}$ was detected (Fig. 4). A transcript of approximately $1700 \mathrm{nt}$ also hybridizes weakly to the nad6 probe. Since the $N r u \mathrm{I}$ probe was gel-isolated from a digest of a $2.0-\mathrm{kb}$ HindIII-PstI subclone that contains part of the $r b c l$ sequence (Fig. 1 A), this signal may represent hybridization to the highly abundant (Gruissem et al. 1988) chloroplast $r b c \mathrm{~L}$ transcripts of this size by a small amount of contaminating $r b c \mathrm{~L}$ DNA in the probe. Consistent with this hypothesis, a probe specific for the $3^{\prime}$ half of the tobacco chloroplast $r b c \mathrm{~L}$ gene hybridizes to the same 1700-nt transcript [data not shown; neither the nad6 nor the $3^{\prime} r b c \mathrm{~L}$ probe hybridize to the larger $r b c \mathrm{~L}$ transcript detected by Makaroff and Palmer (1987)]. However, since the ends of the 750- and 1700-nt transcripts have not been mapped we cannot rule out other possibilities. The size of the major transcript correlates well with the abundant, mitochondrially-derived transcript (\# 18;800 nt) mapped to P3.1 by Makaroff and Palmer (1987).

nad6 is present in a wide range of flowering plant mtDNAs

To determine whether nad6 was present in other plant mitochondrial genomes, the 564-bp NruI probe was used to probe mtDNAs from a wide range of flowering plants 


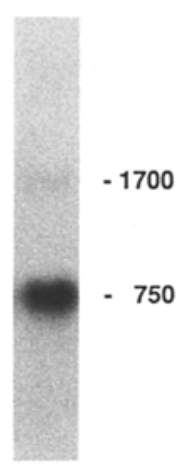

$\operatorname{nad} 6$

Fig. 4. The $B$. campestris nad6 gene produces a single abundant transcript. Northern analysis of the $B$. campestris nad6 locus using a 564 bp $N r u$ I fragment (Fig. 1) as a probe to B. campestris total RNA. Sizes of transcripts are indicated in nucleotides

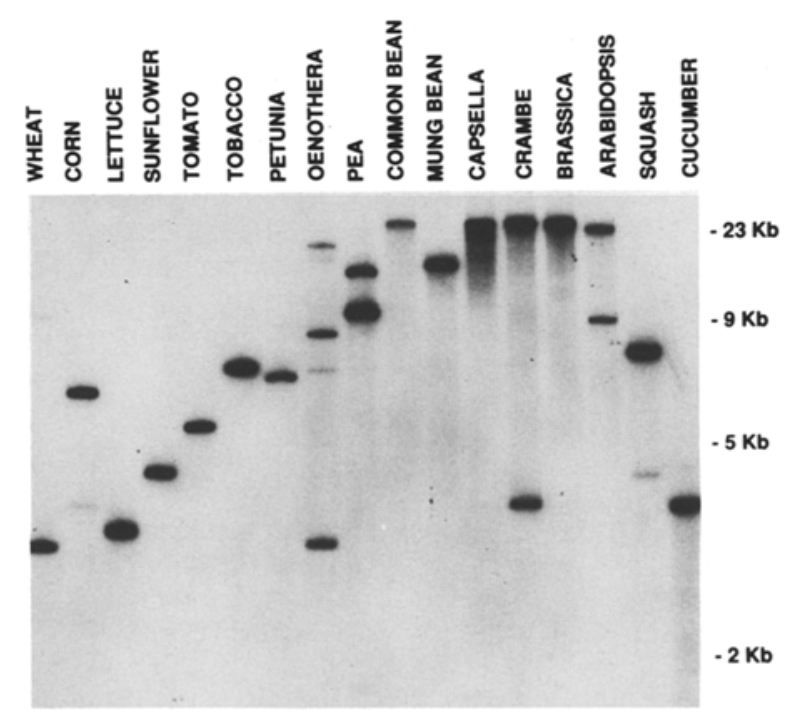

Fig. 5. Nad6 is present in the mtDNA of a wide range of flowering plant species. Hybridization to a filter-blot of $m \mathrm{tDNAs}$ with a 564-bp NruI fragment (Fig. 1) specific to the B. campestris mitochondrial nad6 gene. MtDNAs were cut with restriction enzymes, electrophoresed through a $1.0 \%$ agarose gel and blotted onto a nylon membrane. Wheat (Sall fragments); corn (BamHI); lettuce (SacI); sunflower (SacI), tomato (SalI); tobacco (SalI); Petunia (SaII); Oenothera (SaII); pea (SaII); common bean (SalI); mungbean (SalI); Capsella (SalI); Crambe (SalI); Brassica (Sall); Arabidopsis (SalI); squash ( $S a c I)$; cucumber (Sacl). Other mtDNAs included in the survey but not shown here are watermelon, spinach and radish

(two monocotyledons and 18 dicotyledons). Positive hybridization signals were obtained for all species examined (Fig. 5), demonstrating that the nad6 gene is a conserved feature of flowering plant mitochondrial genomes. Many of the mtDNAs exhibit a single strong hybridizing fragment, indicating that nad 6 is generally a single-copy mitochondrial gene. Several species (Oenothera, pea, Crambe, Arabidopsis) show relatively strong hybridization to more than one fragment both in this experiment and when the mtDNAs are cut with a second restriction enzyme (data not shown), suggesting that part or all of the gene is repeated in these mitochondrial genomes. However, other possibilities, such as disruption of nad6 by cis- or transspliced introns in these organisms, cannot be ruled out. At least three flowering plant nad genes contain exons that are scattered widely in the mitochondrial genome (Chapdelaine and Bonen 1991; Wissinger et al. 1991; Conklin et al. 1991; Knoop et al. 1991; Binder et al. 1992), transcripts of these genes require trans-splicing to produce a functional mRNA.

\section{Discussion}

We have isolated the first gene coding for subunit six of NADH dehydrogenase (nad6) from a flowering plant mitochondrial genome. In $B$. campestris this gene is located on a 3.1-kb Pst I fragment just downstream from a chloroplast-derived $r b c \mathrm{~L}$ sequence. $N a d 6$ produces a single abundant transcript similar in size to a transcript (\#18) mapped to P3.1 by Makaroff and Palmer (1987).

Southern-blot hybridization indicates that an nad6 gene is present in the mitochondrial genome of all of a wide range of flowering plants examined. Although plant mitochondrial protein gene content is generally well conserved, a few genes (three ribosomal protein genes and coxII) are thought to have been lost from the mitochondrion during the evolution of flowering plants (Bland et al. 1986; Wahleithner and Wolstenholme 1988; Schuster et al. $1990 \mathrm{~b}$; Nugent and Palmer 1991). In the case of coxII, the gene loss is tolerated because a functional copy of the gene is present in the nucleus of those legumes lacking the mitochondrial gene (Nugent and Palmer, 1991); presumably this is also the case for the putative ribosomal protein gene losses.

Most plant mitochondrial mRNAs are edited via a cytidine to uridine conversion at various positions along the mRNA molecule (Covello and Gray 1989; Gualberto et al. 1989; Hiesel et al. 1989); more rarely, uridine to cytidine alterations are observed (Gualberto et al. 1990; Schuster et al. 1990a). We do not have a sequence for B. campestris mitochondrial nad6 transcripts or cDNAs and thus do not know whether, or to what extent, the nad6 mRNAs are edited. One way of indirectly inferring editing sites in $B$. campestris nad6 transcripts is to produce an alignment of the $B$. campestris NAD6 protein (deduced from the DNA sequence) and other known NAD6 proteins. Amino-acid positions that are invariant in non-plant mitochondrial proteins but have undergone radical substitutions in the plant mitochondrial protein are likely candidates to be changed by RNA editing (Covello and Gray 1989). Within the most highly conserved region of the NAD6 protein there are at least three amino acids that might be altered as a result of cytidine to uridine editing on $B$. campestris nad6 transcripts to produce a more structurally conserved protein (Fig. 2). Two of these proposed edits involve radical amino-acid changes, Pro(CCA) to Leu(CUA) at position 54, and Ser(UCA) to Leu(UUA) at position 64 . The third possible edited site, at position 57 , does not involve a radical amino-acid change but would alter $\mathrm{His}(\mathrm{CAU})$ to 
Tyr(UAU), which is found at this position in all nonplant mitochondrial nad6 genes.

Seven flowering plant mitochondrial genes are known to contain introns, all 21 of which are group II introns. Three of these split genes (nad1, nad2, nad5) are fragmented into exons that are located far apart from each other on the mitochondrial genome and whose transcripts are joined by trans-splicing (Chapdelaine and Bonen 1991; Binder et al. 1992; Conklin et al. 1991; Wissinger et al. 1991; Knoop et al. 1991). In the case of nad5, trans-splicing integrates an exon of only 22 nucleotides into the mature transcript (Knoop et al. 1991). Direct sequencing of nad6 mRNA or cDNA is needed to determine conclusively whether the gene contains any cisor trans-spliced introns. However, we think it unlikely that the B. campestris nad6 gene contains introns for several reasons. First, we have searched the $B$. campestris nad6 sequence for conserved features of group II introns (Michel et al. 1989). The nad6 sequence shows no similarity to the highly conserved domain V of group II introns. A single region (nucleotides 250-254; Fig. 1) is identical to a conserved sequence found at the $5^{\prime}$ splice site of the A1 subgroup of group II introns (Michel et al. 1989). However, the deduced protein sequence downstream from this region continues to show similarity to the other NAD6 proteins examined (Fig. 2), making it unlikely that splicing occurs at this region. Second, the deduced $B$. campestris protein sequence is reasonably coextensive with Chlamydomonas NAD6; there are no internal gaps of sufficient length to possibly constitute a cis-spliced group II (or group I) intron. Third, both proteins show considerable similarity at their $\mathrm{C}$ - and $\mathrm{N}$-termini (Fig. 2); it is, therefore, highly unlikely that the nad6 mRNA contains a short, terminal, trans-spliced exon that we would have missed. Fourth, the B. campestris nad6 gene exhibits a very simple transcriptional pattern. In general, transspliced genes produce complex transcriptional patterns that reflect the complex organization of the genes (Chapdelaine and Bonen 1991; Wissinger et al. 1991). Fifth, no other region of the $B$. campestris mitochondrial genome hybridizes to a transcript (the presumptive mRNA) of the size produced at this nad6 locus (Makaroff and Palmer 1987).

Since flowering plant mtDNAs are still far from being completely characterized with respect to gene content it remains to be seen whether additional subunits of complex I are coded for by these DNAs. The completely sequenced mitochondrial genome of $M$. polymorpha (186 kb in size; Oda et al. 1992) contains at least seven genes coding for subunits of NADH dehydrogenase. This genome also contains 32 open reading frames whose protein products are so far unidentified. It is possible that some of these ORFs may code for additional subunits of $\mathrm{NADH}$ dehydrogenase and that flowering plants may also contain additional, as yet undescribed, nad genes.

Acknowledgements. We thank M. Hanson, K. Newton and B. Sears for providing several of the mtDNAs used in Fig. 5 and L. Bonen and J. M. Grienenberger for providing unpublished data. This work was supported by NIH grant GM-35087 to J.D.P. and a Midwest Biotechnology Consortium Fellowship to J.M.N.

\section{References}

Attardi G, Schatz G (1988) Annu Rev Cell Biol 4:289-333

Berger S, Ellersiek U, Steinmuller K (1991) FEBS Lett 286:129-132

Binder S, Marchfelder A, Brennicke A, Wissinger B (1992) J Biol Chem 267: 7615-7623

Bland MM, Levings III CS, Matzinger, DF (1986) Mol Gen Genet 204:8-16

Boer PH, Gray MW (1989) Nucleic Acids Res 17:3993

Brennicke A, Moller S, Blanz PA (1985) Mol Gen Genet 198:404410

Chapdelaine Y, Bonen L (1991) Cell 65:465-472

Conklin PL, Wilson RK, Hanson MR (1991) Genes Dev 5:14071415

Covello PS, Gray MW (1989) Nature 341:662-666

Cummings DJ, Domenico JM (1988) J Mol Biol 204:815-839

Cummings DJ, McNally KL, Domenico JM, Matsuura ET (1990) Curr Genet 17:375-402

Dawson AJ, Jones VP, Leaver CJ (1984) EMBO J 3:2107-2113

Devereux J, Haeberli P, Smithies O (1984) Nucleic Acids Res $12: 387-395$

Desjardins P, Morais R (1990) J Mol Biol 212: 599-634

deVries S, Grivell LA (1988) Eur J Biochem 176:377-384

Dupuis A, Skehel M, Walker JE (1991) Biochemistry 30: 2954-2960

Feagin JE, Stuart K (1985) Proc Natl Acad Sci USA 82: 3380-3384

Gass DA, Makaroff CA, Palmer JD (1992) Curr Genet 21:423-430

Gruissem W, Barkan A, Deng XW, Stern D (1988) Trends Genet 4:258-263

Gualberto JM, Wintz H, Weil JH, Grienenberger JM (1988) Mol Gen Genet 215:118-127

Gualberto J, Lamattina L, Bonnard G, Weil JH, Grienenberger JM (1989) Nature 341:660-662

Gualberto JM, Weil JH, Grienenberger JM (1990) Nucleic Acids Res 18: $3771-3776$

Hamby RK, Sims LE, Issel LE, Zimmer EA (1988) Plant Mol Biol Rep 6:175-192

Hiesel R, Wissinger B, Schuster W, Brennicke A (1989) Science 246: $1632-1634$

Jasmer DP, Feagin JE, Stuart K (1985) Mol Cell Biol 5:3041-3047

Knoop V, Schuster W, Wissinger B, Brennicke A (1991) EMBO J 10:3483-3493

Kolodner R, Tewari KK (1972) Proc Natl Acad Sci USA 69: $1830-$ 1834

Koslowsky DJ, Bhat GJ, Perrollaz AL, Feagin JE, Stuart K (1990) Cell 62:901-911

Lamattina L, Grienenberger JM (1991) Nucleic Acids Res 19:3275-3282

Lonsdale DM (1989) The plant mitochondrial genome. In: Marcus A (ed) The biochemistry of plants, vol 15, Academic Press, New York, pp 230-295

Macfarlane JL, Wahleithner JA, Wolstenholme DR (1990) Curr Genet 17:33-40

Makaroff CA, Palmer JD (1987) Nucleic Acids Res 15:5141-5156

Michaelis G, Vahrenholz C, Pratje E (1990) Mol Gen Genet 223:211-216

Michel F, Umesono F, Ozeki H (1989) Gene 82:5-30

Moller IM (1986) Physiol Plant 67:517-520

Newton KJ (1988) Annu Rev Plant Physiol Plant Mol Biol 39: 503 532

Nugent JM, Palmer JD (1988) Curr Genet 14:501-509

Nugent JM, Palmer JD (1991) Cell 66:473-481

Oda K, Yamato K, Ohta E, Nakamura Y, Takemura M, Nozato N, Akashi K, Kanegae T, Ogur Y, Kohchi T, Ohyama K (1992) J Mol Biol 223:1-7

Ohyama K, Fukuzawa H, Kohchi T, Shirai H, Sano T, Sano S, Umesono K, Shiki Y, Takeuchi M, Chang Z, Aota SI, Inokuchi H, Ozeki $H$ (1986) Nature 322: $572-574$

Payne M, Rothwell V, Jasmer DP, Feagin JE, Stuart K (1985) Mol Bio Parasitol 15:159-170

Pritchard AE, Seilhamer JJ, Mahalingam R, Sable CL, Venuti SE, Cummings DJ (1990) Nucleic Acids Res 18:173-180 
Sambrook J, Fritsch EF, Maniatis T (1989) Molecular cloning: a laboratory manual, 2nd ed. Cold Spring Harbor Laboratory, Cold Spring Harbor, New York

Sanger F, Nicklen S, Coulson AR (1977) Proc Natl Acad Sci USA 74:5463-5467

Schuster W, Hiesel R, Wissinger B, Brennicke A (1990a) Mol Cell Biol 10:2428-2431

Schuster W, Wissinger B, Unseld M, Brennicke A (1990 b) EMBO J 9:263-269
Souza AE, Myler PJ, Stuart K (1992) Mol Cell Biol 12:2100-2107 Wahleithner JA, Wolstenholme DR (1988) Nucleic Acids Res 16:6897-6913

Wissinger B, Schuster W, Brennicke A (1991) Cell 65:473-482

Xue Y, Davies DR, Thomas CM (1990) Mol Gen Genet 221:195198

Communicated by D. R. Wolstenholme 